Meta

Journal des traducteurs

Translators' Journal

\title{
Les lectures d'observation : la moisson du traducteur technique
}

\section{Claude Bédard}

Volume 31, numéro 4, décembre 1986

URI : https://id.erudit.org/iderudit/002015ar

DOI : https://doi.org/10.7202/002015ar

Aller au sommaire du numéro

Éditeur(s)

Les Presses de l'Université de Montréal

ISSN

0026-0452 (imprimé)

1492-1421 (numérique)

Découvrir la revue

Citer cet article

Bédard, C. (1986). Les lectures d'observation : la moisson du traducteur technique. Meta, 31(4), 420-427. https://doi.org/10.7202/002015ar d'utilisation que vous pouvez consulter en ligne.

https://apropos.erudit.org/fr/usagers/politique-dutilisation/ 


\section{LES LECTURES D'OBSERVATION : LA MOISSON DU TRADUCTEUR TECHNIQUE}

Tout le monde s'entend sur l'intérêt pour le traducteur technique, outre les recherches visant à trouver des équivalents précis, de faire des lectures d'observation en langue d'arrivée dans le domaine sur lequel porte le texte à traduire. Mais il n'existe que peu d'études qui illustrent ce que le traducteur, concrètement, peut retirer de telles lectures. C'est cette lacune que le présent article veut contribuer à combler, afin notamment de sensibiliser davantage le traducteur à l'utilité de cette démarche et aussi de lui communiquer des indices sur le "comment lire".

Nous présentons ici l'analyse de deux lectures ${ }^{1}$ qui nous ont semblé particulièrement riches en observations, faites à l'occasion d'une traduction sur le reformage catalytique (procédé de raffinage pétrolier). Précisons que notre démarche est celle d'une lecture d'observation, et ne vise pas l'aspect notionne ${ }^{2}$ du vocabulaire : plutôt que de nous interroger sur le sens des mots, nous examinerons leur choix, c'est-à-dire leur côté formel, le domaine du "comment on dit".

Nos observations se regroupent presque d'elles-mêmes en deux grandes catégories : d'une part, les occurrences qui témoignent d'emplois ponctuels, plutôt fermés sur eux-mêmes; d'autre part, les occurrences qui dénotent des tendances débordant les seuls emplois constatés. Dans le premier cas, il s'agit souvent d'emplois spécifiques au domaine technique en cause, tandis que dans le second, on débouche sur une perception générale de la langue technique. 


\section{EMPLOIS PONCTUELS}

Laissant de côté l'ensemble du vocabulaire notionnel (catalyseur multifonctionnel, indice d'octane, réacteur adiabatique...), nous nous intéresserons aux emplois qui facilitent l'expression des énoncés dans lesquels ils se trouvent.

\section{A) COOCCURRENCES}

Le vocabulaire notionnel fait appel, pour son insertion dans le discours, à tout un réseau d'emplois dits de cooccurrences, c'est-à-dire que tel mot appelle de préférence tel ou tel autre mot comme "voisin grammatical ". Voici quelques exemples des cooccurrences relevées.

Adjectifs

... une partie du catalyseur ayant travaillé est remplacée par du catalyseur frais...

... ce qui provoque une formation plus intense de coke...

L'endothermicité élevée de la réformation nécessite...

... ne permet pas de récupération poussée de la chaleur...

Dans ces conditions, qui sont notablement plus sévères que celles...

On remarquera que la grande majorité des adjectifs expriment une idée de degré (beaucoup, peu).

\section{Prépositions}

... qui permettent d'excellentes performances en NO et en rendement...

Avec l'augmentation des exigences en NO...

L'opération consiste à chauffer une essence lourde ... sous une pression de 40 à $50 \mathrm{hpz}$.

... le pourcentage de transformation du cyclohexane en benzène en présence de platine...

Notons que certains mots normalement sans complément dans la langue courante commandent ici une préposition (performances en, exigences en). Toutefois, on peut aussi se demander si ces deux emplois (surtout performances) ne relèvent pas du phénomène du " mot favori ", c'est-à-dire qu'on a cherché à caser le mot dans l'énoncé, quitte à réorganiser celui-ci en conséquence.

\section{Adverbes}

Comme les premiers stades réactionnels sont très endothermiques...

Comme l'ion [PtCl6] ${ }^{-2}$ est fortement chimisorbé...

Ces réactions sont fortement endothermiques...

On note que deux adverbes semblent interchangeables pour modifier endothermique : cela vient illustrer le fait que les cooccurrences, même si elles relèvent des servitudes de la langue, peuvent quand même offrir un certain choix au locuteur.

\section{Verbes}

Les courbes de distillation ASTM portées sur la figure IV.8.14...

... ce qui exalte la réaction parasite de cokage.

... comment les considérations techniques interviennent sur chaque partie...

... le mélange de charge est porté à une température..

L'effluent ... abandonne sa chaleur au gaz recyclé... 
Dans tous ces exemples, le verbe est commandé par son complément - ou par le sujet du verbe passif, ce qui revient au même. Mais cela ne témoigne pas d'une tendance générale.

\section{B) EMPLOIS PARADIGMATIQUES}

Il ne s'agit plus ici de se demander quel mot utiliser avec tel autre, mais quel mot utiliser au lieu de tel autre. Alors que les emplois de cooccurrences revêtent un degré d'obligation assez élevé (ils font largement partie des servitudes de la langue), les emplois paradigmatiques relèvent le plus souvent de préférences; ils contribuent nettement à améliorer la tenue et l'idiomaticité du texte.

\section{Emplois "dans le ton"}

Il s'agit ici notamment de découvrir, parmi des mots de sens a priori équivalent, ceux qui sont "dans le ton " pour le domaine, c'est-à-dire qui ont une certaine saveur idiomatique. On constate en effet que les auteurs du domaine affectionnent certains emplois. Exemples :

... est indispensable pour la marche [le fonctionnement] d'autres unités de la raffinerie...

$\ldots$ du moins jusqu'au voisinage de [à environ] $500^{\circ} \mathrm{C}$.

... on commença à craquer des tonnages [quantités] substantiels de gas-oil...

Les vapeurs de naphta étaient alors dirigées sur [envoyées à] un second réacteur...

Dans cette catégorie se signalent particulièrement certains verbes, à caractère assez général, qui reviennent avec une fréquence notable.

Pour cerner les transformations qui interviennent [se produisent] dans la réformation...

... ce sera la dilution de l'hydrogène par les gaz en $\mathrm{C}_{1}, \mathrm{C}_{2}, \mathrm{C}_{3}$ qui interviendra [aura lieu]. ... pour laisser le temps à la décomposition des hydrocarbures de s'opérer [se faire].

Les verbes s'opérer et intervenir (synonymes entre eux) sont employés dans des situations qui relèvent de la cooccurrence (ils sont appelés par des sujets désignant des phénomènes). Mais il nous semble intéressant de les citer ici en tant que synonymes "relevés" de verbes plus ordinaires comme se produire, avoir lieu, se passer...

... le nom des réactions élémentaires sera précisé pour ce procédé opérant sous pression d'hydrogène.

Seule l'endothermicité ... amène à opérer avec plusieurs réacteurs.

... la gamme des conditions opératoires dans lesquelles travaille industriellement la réformation.

Pratiquement, on travaillera entre des limites qui dépendent...

Les verbes opérer et travailler, employés instransitivement (et avec sujet indéfini ou renvoyant à un procédé), reviennent fréquemment dans le domaine du raffinage.

\section{Ressources de synonymie}

On imagine volontiers le discours technique stéréotypé, voire onocorde. On veut croire, en tous cas, que le vocabulaire y est employé avec une rigueur qui exclut la synonymie. Pourtant, il n'est pas rare de constater une certaine synonymie contrôlée employée simplement pour un motif stylistique de variété.

Si on se rappelle que les essences automobiles ... ont généralement des indices octane compris entre 90 et 100 , on voit que ces coupes n'ont pas... 
Signalons l'entrée d'un personnage qui fait diversion à l'omniprésent pronom " on ".

Les bases dont dispose le raffineur pour constituer les carburants...

Enfin, citons deux groupes de synonymes employés dans le même texte.

charge, matière de charge, matière première

produit liquide, essence, réformat

En somme, l'observation permet de repérer les "emplois favoris", ainsi que de faire provision de synonymes.

\section{C) EMPLOIS RESTREINTS}

Parfois, on constate que l'usage tend à l'emploi systématique d'un mot, à l'exclusion d'autres possibles, dans certaines situations. C'est ainsi que nous avons relevé le verbe chuter (et le substantif chute), employé systématiquement à l'exclusion d'autres synonymes possibles, pour désigner la baisse (abaissement, diminution...) de température dans les réacteurs à cause de la réaction endothermique qui s'y produit.

... un four permet de remonter une température qui a chuté par suite de l'endothermicité.

... dans le premier réacteur, la température peut chuter de 60 à $70^{\circ} \mathrm{C} . .$.

Des termes synonymes (baisser, diminuer, descendre...) sont employés ailleurs dans le texte pour désigner l'évolution d'autres températures.

\section{D) TOURS ABRÉGÉS}

Les textes observés proposent de multiples façons par lesquelles les locuteurs font preuve d'économie. Ces tours abrégés constituent une richesse inestimable pour le traducteur, à la fois pour des raisons de brièveté et d'élégance.

On remarque des procédés assez divers :

Siglaison

On devine déjà que les exigences du $N O$ [nombre d'octanes] et de rendement vont être contradictoires.

Emploi du symbole chimique (ou même de la formule chimique tronquée)

... la transformation est très défavorisée pour les $\mathrm{C}$ [hydrocarbures à six carbones]...

... d'augmenter la production de légers $\left(\mathrm{C}_{1}, \mathrm{C}_{2}, \mathrm{C}_{3}, \mathrm{C} 4\right)$, d'où dilution de 1 ' $\mathrm{H}_{2}$ [hydrogène] de recyclage...

Tournure adjectivale

L'unité comporte un recyclage de l'hydrogène réactionnel [produit par réaction] dont une partie...

... sauf évidemment pour une charge très naphténique [riche en naphtènes]...

Ellipse d'un substantif devant un adjectif

... qu'on évalue à la fois vers davantage de [d'hydrocarbures] légers (hydrocraquage) et davantage de [d'hydrocarbures] lourds (déshydrocyclisation).

... la supériorité du reforming catalytique sur le [reformage] thermique.

Emplois de verbes intransitifs

... on met à l'écart un réacteur ... pour regénérer [le catalyseur] sans arrêter ;

... à partir de chaînes droites nous devrons réformer [la charge] à $500^{\circ} \mathrm{C}$ au moins... 
Emploi avec une préposition normalement inusitée (voir aussi sous 1a)

Le craquage des paraffines lourdes en [pour donner des] paraffines plus légères et branchées...

Cette isooléfine est très rapidement hydrogénée en [pour devenir une] isoparaffine...

Tournure adverbiale (voir aussi sous $2 \mathrm{a}$ )

... la réforme thermique d'un produit déjà réformé catalytiquement [ayant déjà été soumis au reformage catalytique]...

Autres procédés

... mais une base d'essence destinée à être mélangée avec d'autres bases pour obtenir une essence aux [respectant les] spécifications.

... une bonne partie du gain d'octane [amélioration de l'indice d'octane] constaté dans le réformat...

Aromatiques formés dont ex-paraffines [anciennes paraffines]..

Comme on peut le constater, les procédés d'abrègement sont nombreux et confèrent souvent au texte une certaine idiomaticité.

\section{TENDANCES}

Un certain nombre d'emplois renseignent le traducteur non seulement sur euxmêmes, mais, observés en nombre suffisamment important, permettent de déduire intuitivement des phénomènes plus larges. C'est ainsi que nous avons pu dégager de ces lectures deux tendances fort intéressantes.

\section{DÉRIVATION}

La dérivation est certes un mécanisme fondamental de la langue. Mais il se trouve qu'en technique, et dans chaque domaine particulier, il s'en fait un usage qui peut surprendre : en effet, on est habitué à reconnaître certaines limites à la dérivation - au-delà de celle qui s'exprime effectivement dans l'usage général et que consignent les dictionnaires, il y a la dérivation virtuelle, que le locuteur soucieux de correction langagière hésite à actualiser.

Or, les textes observés montrent fort peu de gêne à entraîner les mots hors de leur catégorie grammaticale première. En fait, c'est exactement ce qui se produit dans la langue générale; mais cette dérivation est lexicalisée depuis très longtemps, alors que ce n'est guère le cas en technique.

Adjectifs

Comme les premiers stades réactionnels [de réaction] sont très endothermiques...

... il est bon de donner ses caractéristiques opératoires [d'exploitation] essentielles pour...

... la mise au point de catalyseurs plus sélectifs, moins cokants [générateurs de coke] « in situ».

On remarque deux adjectifs de grande consommation dans le domaine du raffinage : réactionnel et, surtout, opératoire; ce dernier, dérivé sûrement de l'anglais operating, n'en est pas moins tout à fait implanté dans l'usage. Notons aussi les terminaisons en -ant et en -able, qui semblent accolées librement aux adjectifs les plus divers. 
Adverbes

... puisque les conditions sont thermodynamiquement favorables.

... on conçoit de ce fait la possibilité d'obtenir préférentiellement une isooléfine.

Cinétiquement, elle a été étudiée dans certains cas.

Malgré leur aspect massif, les adverbes sont plus directs que les tours « du point de vue thermodynamique " ou "de façon sélective ". On peut certes y déceler l'influence de l'anglais, mais cela ne suffit pas à les désavouer.

Verbes

... en général, on cyclise difficilement plus de $20 \% \ldots$

L'abaissement du P.I. correspond à la fraction hydrocraquée...

Les $\mathrm{C}_{5}$ et les $\mathrm{C} 6$ sont isomérisés dans des unités spécifiques...

Comme l'ion [PECl6] ${ }^{2}$ est fortement chimisorbé...

Un nombre impressionnant de verbes sont dérivés de substantifs (cyclisation, hydrocraquage, isomérisation, chimisortion) qui décrivent un processus ; ces emplois, qui peuvent surprendre au premier contact, sont pourtant parfaitement naturels et constituent souvent le seul moyen d'éviter une périphrase.

Substantifs

... qui donne la "réformabilité » de cette charge.

... sont surtout remarquables par une régénérabilité intégrale.

Le suffixe -abilité permet d'éviter la tournure plus longue aptitude à la...

De façon générale, on peut dire que la motivation qui expliquerait cette "soif de dérivation" est l'économie : généralement, le changement de catégorie grammaticale permet d'adopter une formulation plus brève.

B) EMPLOIS VIVANTS

Enfin, si l'on croit que le discours technique est terne et sans vie, nos lectures font vite changer d'avis. On peut relever en effet plusieurs emplois qui dénotent au contraire un certain goût pour la fantaisie et la vigueur d'expression. Nous avons distingué quatre catégories.

\section{Animisme}

Il s'agit d'associer l'univers des choses à celui des êtres vivants ${ }^{3}$. Soulignons que la plupart des emplois animistes ne visent pas particulièrement a faire image, mais dénotent plutôt une tendance presque inconsciente chez le locuteur à "voir » dans les choses une matière vivante.

... la réformation catalytique sera amenée à se surpasser dans la production...

... la thermodynamique serait justiciable de la figure III.8.6...

... mais les intermédiaires oléfiniques ... sont défavorisés par une basse température...

La courbe ASTM du réformat a évidemment intérêt à...

Voyons maintenant comment se comportent à ce point de vue les essences...

Cette réaction ... s'accommode donc de vitesses spatiales élevées. 


\section{Mots fantaisistes}

Ici, le locuteur cherche délibérément à faire image. Ce procédé, qui a l'avantage de relancer l'attention du lecteur, témoigne aussi de l'intérêt que porte le locuteur au sujet traité.

... les aromatiques constituent «l'or d'un réformat », du moins pour les fractions assez lourdes...

Les conditions opératoires gravitent autour des valeurs suivantes :

... seront peut-être obligés de les envisager si la course à l'octane...

\section{Mots émotifs}

Parfois, le locuteur emploie des mots à consonance émotive. Ces emplois peuvent surprendre si l'on croit que la neutralité est de rigueur pour les textes techniques.

... ce qui est très gênant en raison de la formation parallèle d'aromatiques...

... puis provoque une chute catastrophique des performances...

Ces réactions sont intéressantes, car elles améliorent...

\section{Tours naturels}

Enfin, on n'hésite pas à l'occasion à adopter des tournures qui relèvent de l'expression générale, spontanée. On constate donc que le discours technique n'est pas cantonné à une langue aseptisée, mais accepte - voire recherche - des tournures qui sonnent « humain».

Par contre, la faible vitesse est un lourd handicap qui oblige à forcer les conditions opératoires...

... aux températures élevées elle prend le pas sur l'isomérisation...

Dans le second réacteur, l'endothermicité l'emporte encore largement...

La propension à "épicer" son discours de tours naturels varie d'un auteur à l'autre; en outre, selon le genre de texte, celle-ci soit devra s'effacer complètement (notice technique), soit pourra se donner libre cours (article de vulgarisation). Mais il faut reconnaître son existence et son potentiel pour le traducteur.

\section{CONCLUSION}

L'observation de textes en langue d'arrivée permet donc au traducteur de faire une moisson intéressante d'emplois individuels pertinents au domaine, qui lui permettent de s'exprimer de façon non seulement correcte, mais aussi pleinement idiomatique.

Mais il y a une autre conséquence, peut-être plus importante encore : le traducteur qui lit sur le sujet en langue d'arrivée gagne un aplomb rédactionnel certain. Grâce à celui-ci, au lieu de simplement réagir à la formulation du texte à traduire, il sera porté davantage à s'exprimer de façon spontanée en LA. C'est là un ingrédient essentiel d'une traduction de qualité.

par ailleurs, la lecture de textes techniques permet au traducteur de saisir à la longue ce qui caractérise la langue technique dans sa globalité. À force de fréquenter celleci, le traducteur saura mieux comment "oser " s'exprimer, quel que soit le domaine technique en cause ${ }^{4}$.

Précisons toutefois que la langue technique n'évolue pas de façon homogène d'un domaine à l'autre. Dans le cas présent, les textes étaient tirés du domaine pétrolier, domaine relativement récent et qui n'est donc pas tributaire d'usages centenaires. Celui-ci, comme tous les domaines de pointe, se caractérise par un dynamisme d'expression relativement grand - inspiré en partie par une certaine influence de l'anglais (on relève dans les deux textes divers emplois douteux inspirés vraisemblablement par cette langue). 
Par contre, cette hétérogénéité est justement un facteur d'évolution et d'enrichissement. Les domaines techniques individuels (surtout les plus avant-gardistes) exercent une certaine influence non seulement sur les tendances générales de la langue technique, mais aussi sur l'ensemble des moyens ponctuels d'expression à la façon de ces innombrables usages régionaux qui ont nourri la langue commune. Aussi, par exemple, la tendance à la tournure adverbiale (de plus en plus répandue dans l'usage technique général), a vraisemblablement pris naissance dans certains domaines soumis à une forte influence anglo-saxonne - notamment le pétrole. De même, on peut soupçonner que l'adjectif opératoire (voir 2a), maintenant employé dans dés domaines divers, est entré dans l'usage par la porte du domaine pétrolier.

Enfin, signalons que le mode d'expression, surtout sur le plan stylistique, peut varier d'un auteur à l'autre, et aussi d'un genre de texte à l'autre. Ainsi, la tendance décrite en $2 \mathrm{~b}$ se manifeste plus fortement dans le premier texte (Montarnal et Duhaut) que dans le second. Par ailleurs, celle-ci est à encourager dans un article de revue industrielle, mais à restreindre dans une notice technique.

En somme, par-delà les avantages immédiats qu'il en retire pour la traduction d'un texte précis, le traducteur technique a intérêt à faire le plein d'observations générales qui enrichiront son bagage de moyens d'expression et qui l'aideront à en doser l'emploi.

Claude BÉDARD

Traducteur, Montréal, Canada

Notes

1. R. Montarnal et P. Duhaut (1972) : "Réformation catalytique ", dans Pierre Wuithier (dir.), le Pétrole Raffinage et génie chimique, t. II, Paris, Technip, pp. 775-810.

Xavier Normand (1968) : Leçons sommaires sur l'industrie du raffinage du pétrole, t. I, Paris, Technip, pp. 275-298.

2. Pour plus de précisions sur les différentes catégories de vocabulaire (notionnel, formel, syntagmatique, paradigmatique...) évoquées ici, voir Claude Bédard (1986) : la Traduction technique - Principes et pratique, Montréal, Linguatech, chap. 6.

3. Voir Claude Bédard (1985) : "Quand la langue prête vie aux choses", dans Circuit, no 10, septembre.

4. Une analyse détaillée des particularités de la langue technique figure au chap. 7 de l'ouvrage citéà la note 2. 